\title{
Sistema Multiagente para Apoiar a Realização de Atividades Locativas em Ambientes Virtuais de Aprendizagem
}

\author{
Wesley de S. Viana, Elaine H. T. Oliveira, Wagner Gaspar \\ Instituto de Computação - Universidade Federal do Amazonas (UFAM) \\ Caixa Postal 69077 - 000 - Amazonas - AM - Brasil \\ \{wesley, elaine, wg\}@icomp.ufam.edu.br
}

\begin{abstract}
In recent years, the rapid growth in use of mobile devices has boosted the development of new tools and technologies in order to provide conditions for use in various educational contexts. Given this scenario, this work presents a recent technology for virtual learning environments involving multi-agent system, location-aware mobile learning and for creating and conducting activities restricted by location.
\end{abstract}

Resumo. Nos últimos anos, o crescimento acelerado na utilização de dispositivos móveis impulsionou o desenvolvimento de novas ferramentas $e$ tecnologias com intuito de oferecer condições para utilização em diversos contextos educacionais. Diante desse cenário, este trabalho apresenta uma tecnologia recente para ambientes virtuais de aprendizagem envolvendo sistema multiagente, reconhecimento de localização e aprendizagem móvel para criação e realização de atividades restrita por localização.

\section{Introdução}

Atualmente, o crescimento do ensino à distância $(\mathrm{EaD})$, contribuiu para a inserção de uma maior parcela da população nessa modalidade de ensino. De acordo com o Instituto Nacional de Estudos e Pesquisas Educacionais Anísio Teixeira (INEP, 2013), entre os anos de 2011 e 2012, as matrículas em cursos à distância cresceram 12,2\%, enquanto que nos cursos presenciais, o aumento foi de 3,1\% [Portal MEC, 2013]. Esse crescimento é justificado pela facilidade de acesso oferecida por essa modalidade do ensino, tais como: flexibilidade de horário, redução de custos, entre outros.

Uma das formas que potencializa o ensino é através dos dispositivos móveis, uma modalidade de ensino também conhecida como Mobile Learning. Os dispositivos móveis nos últimos anos se tornaram mais presentes no cotidiano das pessoas, além de possuírem um grande potencial no ensino a distância [Ally, 2009]. Seguindo o mesmo raciocínio, de acordo com [Nunes et al, 2012], os AVAs são considerados como uma opção tecnológica para atender as novas necessidades educacionais. Dessa forma, a junção com os dispositivos móveis e seus recursos tecnológicos permitem maiores possibilidades no desenvolvimento de novas tecnologias educacionais.

O uso da internet e dos dispositivos móveis proporcionam novas formas de ensino e aprendizagem. Hoje em dia, é comum as pessoas utilizarem os dispositivos móveis para acessar a internet, ler informações ou até mesmo aprender conteúdos 
essenciais para melhorar o desempenho acadêmico. Neste sentido, este artigo aborda conceitos de mobilidade, reconhecimento de localização para possibilitar a criação e realização de atividades locativas, ou seja, atividades que necessitam de localização para a sua realização e o desenvolvimento de um sistema multiagente em AVAs para apoiar a realização dessas atividades.

O artigo está organizado da seguinte forma: na seção 2 é apresentado os conceitos relacionados, abordando o conceito de agentes, mobile learning $\mathrm{e}$ reconhecimento de localização (location awareness). Na seção 3 , é abordado a arquitetura de sistema, na seção 4 temos as conclusões e trabalhos futuros. Por fim, na seção 5 temos os agradecimentos e na seção 6 as referências.

\section{Conceitos Relacionados}

Diversos conceitos foram utilizados nesse trabalho para fundamentar a pesquisa, um desses conceitos é os de agentes, que podem ser considerados uma entidade de software que funcionam de maneira autônoma e independente, a fim de atingirem os seus objetivos [Wooldridge, 2009]. Sendo capazes de perceber o seu ambiente por meio dos sensores e de agir sobre esse ambiente através dos atuadores [Russel e Norvig, 2002]. Além disso, podem se comunicar com outros agentes para atingir um objetivo, ampliando as possibilidades de funcionamento.

Existem várias pesquisas abordando o uso de agentes para desempenhar funções em AVAs, seja para: recomendar conteúdo [Silva et al, 2011], acompanhar atividades no ambiente [Alencar e Netto, 2012], monitorar informações em fórum [Júnior et al, 2011] e diversas outras funcionalidades, inclusive pode ser utilizado na área de reconhecimento de localização. Diante desse número de possibilidades, se torna extremamente importante a sua utilização em ambientes virtuais de aprendizagem.

A área de Mobile Learning é uma área que trata da utilização de dispositivos móveis para apoiar o processo de ensino aprendizagem. Essa mobilidade é possibilitada por meio de aplicação e tecnologias da informação móveis e sem fio (TMSF), tais como: palmtops, laptops, smartphones, dentre outros. O uso de TMSF, aliado à interação com atores humanos distantes geograficamente e fisicamente, pode propiciar a ocorrência de processos de ensino e de aprendizagem [Graziola, 2009]. O aplicativo Moodle Mobile [Moodle Mobile, 2014], é um exemplo de software que contribui para o processo de ensino aprendizagem, promovendo a mobilidade de ensino e permitindo ao usuário acessar informações em qualquer hora e em qualquer lugar.

$\mathrm{Na}$ área de reconhecimento é definida como a capacidade de um sistema reagir de acordo com contexto de localização do usuário [Berman et al, 2008]. Essa é uma área de pesquisa que está preocupada com informações de contexto por localização, seja ela por GPS, localização indoor ou por triangulação. Os dispositivos são ferramentas tecnológicas com diversos sensores como o GPS, WiFi, 3G, NFC entre outros que favorece a utilização técnicas de reconhecimento de localização.

\section{Arquitetura de Sistema}

A arquitetura foi desenvolvida buscando incluir agentes com a finalidade de apoiar os estudantes na realização de atividade restrita por localização. As funções desempenhadas pelos agentes são: manter os alunos informados sobre as tarefas e 
prazos, além de ajudar os estudantes no trajeto até os locais de atividade através do envio dessas informações por SMS e e-mail. A arquitetura é composta pelo estudante que realiza a atividade e o pelo professor que elabora as tarefas locativas dentro do ambiente Moodle. A Figura 1 mostra a arquitetura do sistema.

$\mathrm{Na}$ arquitetura proposta foi utilizado o plugin de modificação de curso format_gps [Kamedia, 2013] para permitir a elaboração e realização de atividades com restrição por localização. Foram feitas algumas alterações para melhorar a utilização e permitir o funcionamento dos agentes. No envio de SMS foi utilizado um modem 3G com o NowSMS [NowSMS, 2014]. Para o desenvolvimento dos agentes foi utilizado o JADE [Java Agent Development Framework, 2013], um software livre com licença LGPL, desenvolvido em JAVA. Também foi utilizado o padrão FIPA (Foundation for Intelligent Physical Agents) para a comunicação entre agentes.

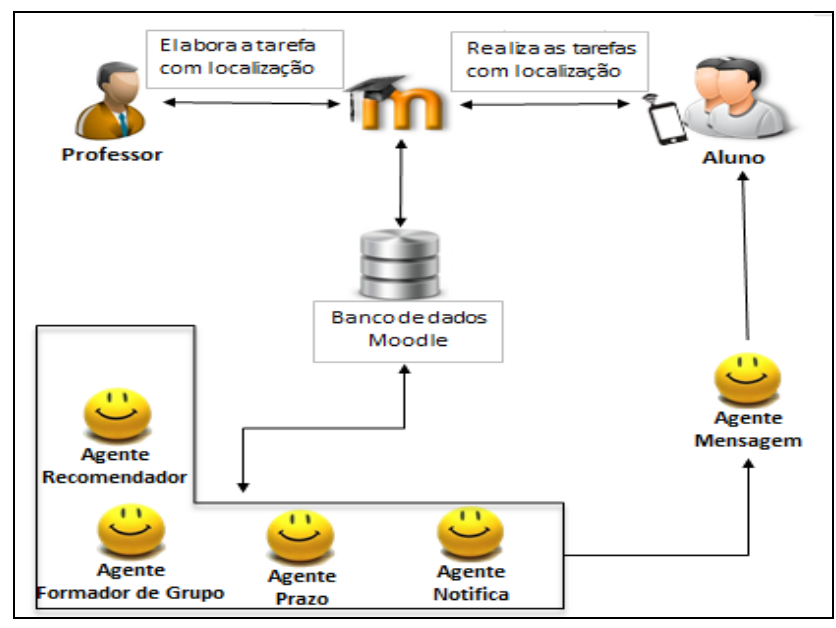

Figura 1. Arquitetura Multiagente.

No módulo de elaboração de atividades do plugin, o professor restringe uma tarefa por localização, conforme a Figura 2. No passo seguinte, o aluno utiliza o dispositivo móvel com GPS e internet e realiza a atividade no local definido conforme a Figura 3 e 4.

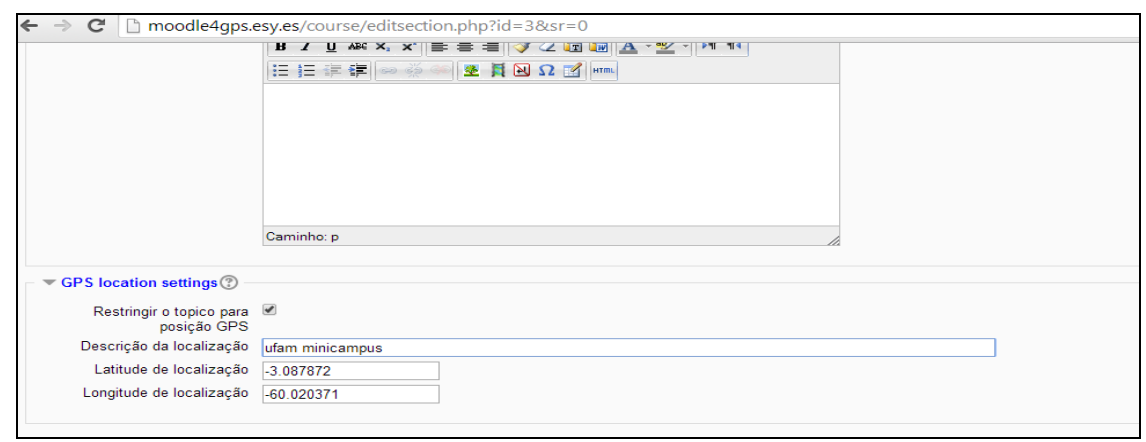

Figura 2. Restringindo uma atividade por localização

$\mathrm{Na}$ Figura 2, temos a restrição de um tópico no Moodle por localização (longitude e latitude), informações disponíveis no Google Maps. Ao restringir por localização, o usuário só visualizará a atividade caso esteja em um raio de até 50 metros da localização definida. 


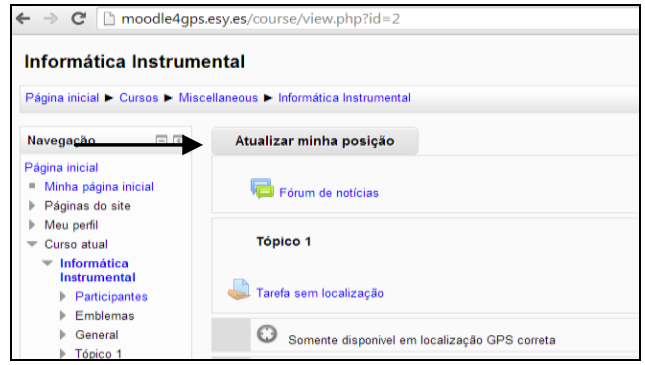

Figura 3. Visualiza atividades

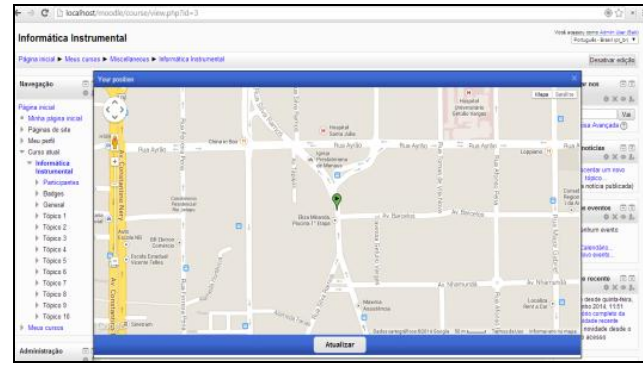

Figura 4. Reconhece a localização

Na Figura 3, temos um curso com uma atividade locativa. A seta mostra o local para atualizar a posição do usuário. Ao atualizar, o ambiente reconhece e verifica se o mesmo se encontra em algum local de atividade, conforme a Figura 4, caso o usuário esteja no perímetro de uma atividade ele visualiza o conteúdo e realiza a atividade. Abaixo, temos o papel desempenhado pelos agentes dentro do ambiente Moodle:

Agente Notifica: Responsável por verificar a postagem de novas atividades locativas e fornecer a descrição do local de realização da atividade e um link com uma rota para chegar ao local de atividade.

Agente Prazo: Disponibiliza algumas informações com relação a prazo de encerramento de tarefa: título, data de fim e um link para acesso a rota ao local de atividade.

Agente Mensagem: Estabelece a conexão com o modem 3G e envia o SMS e email para os alunos com o conteúdo fornecido pelos outros agentes.

Agente Recomendador: Caso o usuário acesse o ambiente e não esteja no raio de atividade ele recomenda a mais próxima da posição atual do usuário informando o título, a distância para o local de atividade e o link de rota de acesso.

\section{Conclusões e trabalhos futuros}

Este artigo apresentou uma pesquisa sobre a utilização de reconhecimento de localização, dispositivos móveis com apoio de agentes em um AVA, para elaborar e realizar atividades com localização pré-definidas.

Durante a pesquisa, foi incluído como trabalho futuro o cadastro de preferência de funcionamento de agentes, esse módulo de software constitui de regras para funcionamento dos agentes. Outro modulo de software também será desenvolvido, onde será possível visualizar os locais de atividades em um mapa, e por fim outro ponto a se destacar é a inclusão de um aplicativo móvel baseado no Moodle Mobile, o aplicativo oficial do Moodle de código fonte aberto para o desenvolvimento de funcionalidades. $\mathrm{O}$ objetivo do desenvolvimento do aplicativo é facilitar o acesso a funcionalidade do proposto trabalho.

\section{Agradecimentos}

Partes dos resultados apresentados neste trabalho foram obtidos através de atividades de R\&D do projeto de "Grande escala programa de qualificação em tecnologias móveis PROMOBILE", patrocinado pela Samsung Eletrônica da Amazônia Ltda. nos termos da lei federal $n^{\circ} 8.248 / 91$. 


\section{Referências}

Alencar, M. A. D. S. e Netto, J. F. de M. (2012). "Sistema Multiagente para Apoiar a Percepção e o Acompanhamento de Atividades em Ambientes Virtuais de Aprendizagem". Anais do XXIII Simpósio Brasileiro de Informática na Educação (SBIE). Rio de Janeiro - RJ.

Ally, M. (Ed.). (2009). "Mobile Learning: Transforming the Delivery of Education and Training". Editora AU Press, Athabasca University Press. ISBN: ISBN 978-1-89742544-2.

Berman, A. M., Lewis, S. M. e Conto, A. (2008) "Location-Aware Computing". November 2008. URL: < http://net.educause.edu/ir/library/pdf/DEC0803.pdf/>.

Graziola, P. (2009). “Aprendizagem com Mobilidade (M-Learning) nos Processos de Ensino e de Aprendizagem: Reflexões e Possibilidades. Novas Tecnologias na Educação (CINTED), Vol 7, n.1.

Jade. (2013) “Java Agent Development Framework”. URL: < http://jade.tilab.com/>.

Júnior, R. L. de O., Esmin, A. A. A., Coelho, T. A., Araujo, D. L., Silva, L. A. e Giroto, R. (2011). "Uma Ferramenta de Monitoramento Automático de Mensagens de Fóruns em Ambientes Virtuais de Aprendizagem”. Anais do XXII Simpósio Brasileiro de Informática na Educação (SBIE). Aracaju-SE.

Kamedia. (2013) "Plugin Kamedia GPS Format Free". URL $<$ http://blog.kamedia.de/index.php/2013/07/02/the-new-moodle-gps-course-format/ >.

Moodle. (2014) "A Free, Open Source Course Management System for Online Learning”. URL: <http://www.moodle.org/>.

Moodle Mobile. (2013) “Aplicação Moodle Mobile". URL: $<$ http://docs.moodle.org/27/en/Mobile_app/>.

Nunes, C. S., Torres, M. K. L.; de Oliveira, P. C. e Nakayama, M. K. (2012) "O Ambiente Virtual de Aprendizagem Moodle: Recursos para os Processos de Aprendizagem Organizacional". Anais do XXIII Simpósio Brasileiro de Informática na Educação (SBIE). Rio de Janeiro - RJ.

Portal Ministério da Educação - MEC. (2013). URL: $<$ http://portal.mec.gov.br/index.php?option=com_content\&view=article\&id=19077:c enso-aponta-aumento-de-44-e-matriculas-superam-7-milhoes-\&catid=212\&Itemid/>

Russel, S. e Norvig, P. (2002) “Artificial Intelligence: A Modern Approach". New Jersey, Prentice Hall.

Silva, L. C. M., Neto, F. M. M. e Junior, L. J. (2011) "MobiLE: Um Ambiente Multiagente de Aprendizagem Móvel para Apoiar a Recomendação Sensível ao Contexto de Objetos de Aprendizagem". XXII Simpósio Brasileiro de Informática na Educação (SBIE). Aracaju - SE.

Wooldridge, M. (2009) “An Introduction to Multiagent Systems”. Ed. Wiley, England, Second Edition. 